\title{
Capsaicin Induces Apoptosis in Human Small Cell Lung Cancer via the TRPV6 Receptor and the Calpain Pathway
}

\author{
Jamie K. Lau ${ }^{1}$, Kathleen C. Brown ${ }^{1}$, Aaron M. Dom ${ }^{1}$, Theodore R. Witte ${ }^{2}$, Brent A. \\ Thornhill $^{1}$, Clayton M. Crabtree ${ }^{1}$, Haley E. Perry ${ }^{1}$, J. Michael Brown ${ }^{1}$, John G. Ball ${ }^{1}$, \\ Rebecca G. Creel ${ }^{1}$, C. Luke Damron ${ }^{1}$, William D. Rollyson ${ }^{1}$, Cathryn D. Stevenson ${ }^{1}$, W. \\ Elaine Hardman ${ }^{2}$, Monica A. Valentovic ${ }^{1}$, A. Betts Carpenter ${ }^{3}$, and Piyali Dasgupta ${ }^{1,{ }^{*}}$ \\ ${ }^{1}$ Department of Pharmacology, Physiology and Toxicology, Joan C. Edwards School of \\ Medicine, Marshall University, Huntington, WV, USA \\ 2 Department of Biochemistry and Microbiology, Joan C. Edwards School of Medicine, Marshall \\ University, Huntington, WV, USA \\ ${ }^{3}$ Department of Anatomy and Pathology, Joan C. Edwards School of Medicine, Marshall \\ University, Huntington, WV, USA
}

\section{Abstract}

Capsaicin, the pungent ingredient of chili peppers, displays potent anti-neoplastic activity in a wide array of human cancer cells. The present manuscript examines the signaling pathways underlying the apoptotic activity of capsaicin in human small cell lung cancer (SCLC) in vitro and in vivo. Studies in neuronal cells show that capsaicin exerts its biological activity via the transient receptor potential vanilloid (TRPV) superfamily of cation-channel receptors. The TRPV family is comprised of six members (TRPV1-6). Capsaicin is an agonist of the TRPV1 receptor. We observed that capsaicin-induced apoptosis in human SCLC cells was mediated via the TRPV receptor family; however it was independent of TRPV1. Surprisingly, the apoptotic activity of capsaicin required the TRPV6 receptor. Depletion of TRPV6 receptor by siRNA methodology abolished the apoptotic activity of capsaicin in SCLC cells. Immunostaining and ELISA showed that TRPV6 receptor was robustly expressed on human SCLC tissues (from patients) and SCLC cell lines but almost absent in normal lung tissues. This correlates with our results that capsaicin induced very little apoptosis in normal lung epithelial cells. The proapoptotic activity of was mediated by the intracellular calcium and calpain pathway. The treatment of human SCLC cells with capsaicin induced increased the activity of calpain 1 and 2 by three-fold relative to untreated SCLC cells. Such calpain activation, in response to capsaicin, was downstream of the TRPV6 receptor. Taken together, our data provide insights into the mechanism underlying the apoptotic activity of capsaicin in human SCLCs.

*dasgupta@marshall.edu.

The authors declare that they have no conflict of interest 


\section{Introduction}

Small cell lung cancer (SCLC) is a neuroendocrine tumor characterized by early dissemination and rapid metastasis (1). It represents $13 \%$ of all lung cancer cases and is the most aggressive form of lung cancer, with an overall 5-year survival time of less than 5\% (2). Such sobering facts define the arena where novel therapeutic interventions are urgently needed to combat this lethal malignancy. Recent studies have shown that dietary compounds by themselves or in combination with standard chemotherapy can suppress the growth of several human cancers (3-5). The identification of dietary compounds that can decrease the growth of SCLCs could foster the hope of novel therapies for this disease.

Capsaicin (trans-8-methyl-N-vanillyl-6-noneamide) is the pungent ingredient of chili peppers (6) (7). Chemoprevention experiments in mice and rats show that capsaicin abrogates carcinogenesis of the skin, colon, lung, tongue and prostate (8-11). Recent studies have explored the applications of capsaicin as a potential agent for cancer therapy. Several convergent studies have revealed that capsaicin caused G1/G0 cell cycle arrest in human epidermoid carcinoma, prostate carcinoma, SCLC and breast cancers in both in vitro and in vivo model systems $(7,12)$. In addition, high concentrations of capsaicin trigger apoptosis in several types of human cancer cells, including breast cancer and gastric cancer $(13,14)$. Similarly, long-term exposure to capsaicin for (36-72 hours) also produces robust apoptosis in non-small cell lung cancer (NSCLC), T-cell leukemia, esophageal carcinoma, astroglioma, prostate and colon cancer cells in cell culture models $(7,15)$. Most importantly, the anti-neoplastic activity of capsaicin was found to be specific towards cancer cells, leaving normal cells relatively unaffected.

The ability of capsaicin to produce a "heat-sensation" is mediated by the transient receptor potential vanilloid 1 (TRPV1) receptor which belongs to the Transient Receptor Potential (TRP) superfamily of cation-channel receptors (16). The TRPV receptor family is comprised of six members (TRPV1-6). Data from Caterina et al., (1997) showed that capsaicin is a high affinity agonist of the TRPV1 receptor. The growth-inhibitory activity of capsaicin in human cancer cells involves multiple mechanisms like induction of reactive oxygen species (ROS) and peroxynitrite, p53 stabilization, inhibition of NADH oxidase activity, ERK, EGFR and HER-2, c-Jun $\mathrm{NH}_{2}$-terminal kinase, nuclear factor- $\mathrm{\kappa B}$, activator protein-1, and mitochondrial respiration $(7,15)$.

The apoptotic activity of capsaicin in androgen-independent $\mathrm{PC}-3$ prostate cancer cells, gliomas and urothelial cancer cells has been found to be mediated via the TRPV1 receptor (17-19). The binding of capsaicin to the TRPV1 receptor induced the stimulation of $\mathrm{p} 38$ MAP kinase, which increased mitochondrial permeability and caused downstream activation of caspase-3, leading to cellular apoptosis (18). In urothelial cancers, capsaicin caused clustering of TRPV1 receptors, stimulating activation of the Fas pathway via ATM kinase (17). Capsaicin has been found to induce degradation of the Fas associated factor FAF-1, which sensitizes cells to apoptotic cell death. A few studies have addressed the effect of capsaicin on other proteins of the TRPV receptor family. Out of the six members of the TRPV family, capsaicin has been shown to regulate the function of the TRPV6 receptor. The rest of TRPV receptors are not responsive to capsaicin. 
The mechanisms underlying the apoptotic activity of capsaicin in human SCLC has not been investigated. The apoptotic activity of capsaicin was observed in human SCLC cell lines as well as in human SCLC cells xenografted in nude mouse. The pro-apoptotic effects of were dependent on the TRPV receptor family but was not dependent on TRPV1. Surprisingly, we observed that capsaicin-induced apoptosis was mediated by TRPV6. The levels of TRPV6 were found to be high in human SCLCs and low in normal lung epithelium. We believe that such difference in expression of TRPV6 could at least, in part explain the specificity of capsaicin for lung cancer cells versus normal lung cells. The apoptotic activity of capsaicin was mediated by the calpain pathway, specifically calpain- 1 and calpain- 2 . When human SCLC cells were treated with capsaicin, we observed an elevation in calpain-1 and calpain-2 activity. The activation of calpain (upon treatment of capsaicin) was downstream of the TRPV6 receptor in human SCLCs. Taken together, our data suggest that capsaicin displays apoptotic activity in human SCLC cells via the TRPV6 and downstream calpain pathway. Furthermore, our data reveal that TRPV6 may be a useful molecular target for therapy of human SCLC.

\section{Materials and methods}

\section{Ethics statement}

All procedures involving nude mice were conducted according to the Animal Care and Use guidelines in a facility fully accredited by the Association for Assessment and Accreditation of Laboratory Animal Care (AAALAC) International and were approved by the Institutional Animal Care and Use Committee (IACUC) of Joan C. Edwards School of Medicine, Marshall University (protocol \#371).

\section{Cell Lines and culture}

The human SCLC cell lines NCI-H82, NCI-H69 (hereafter referred to as H82 and H69), DMS 53 and DMS 114 were obtained from American Type Culture Collection (Rockville, MD, USA). They were cultured as described in Supplementary Methods. The cell lines were authenticated by the ATCC Cell Authentication service using Short Tandem Repeat (STR) profiling techniques (data not shown).

Primary normal human bronchial epithelial cells (NHBE) and small airway epithelial cells (SAEC) were obtained from Lonza Technologies (Switzerland) and cultured according to the manufacturer's instructions. These cells were authenticated by Lonza and a certificate of analysis was provided. All experiments using NHBEs and SAECs were performed between passages 3-8 (20).

\section{Measurement of caspase-3 activity}

DMS 53 human SCLC cells were cultured to 70\% confluence as described above. On the day of the assay, the medium of the cells was changed to Waymouth's medium containing $1 \%$ FBS. Subsequently, cells were treated with the varying concentrations of capsaicin for 36 hours at $37^{\circ} \mathrm{C}$. Cell Lysates were made using the Caspase-3 Activity Kit (EMD Millipore Corporation, Billerica, MA, USA). An aliquot of the cell lysate containing one hundred micrograms of protein used for the measurement of caspase- 3 activity, according to the 
manufacturers' protocol. Cells treated with $10 \mu \mathrm{M}$ cisplatin were used as the positive controls for the assay.

Each sample was measured in duplicate and the whole experiment was performed two independent times. Caspase- 3 activity in untreated lysates was considered to be equal to 1 , and the activity observed in treated lysates was calculated as fold increase relative to the untreated control sample. The experimental procedure was identical in DMS 114, H69 and H82 cells.

\section{Cleaved PARP ELISA assay}

The human SCLC cell line DMS 53 was grown to $70 \%$ confluence in $100 \mathrm{~mm}$ tissue culture dishes. On the day of the experiment, the medium of the cells was changed to Waymouth's medium containing $1 \%$ FBS. The cells were treated with the indicated concentration of capsaicin for 36 hours at $37^{\circ} \mathrm{C}$. Lysates were prepared using the protocol as described above. One hundred microgram of lysate was used for each sample. The amount of cleaved PARP in each sample was measured by Cleaved PARP ELISA assay (Cell Signaling, Danvers, MA), according to manufacturers' protocol. The absorbance value of control untreated cell was taken as 1, and the absorbance of capsaicin-treated cells were graphically represented as fold-increase relative to the control. Cells treated with $10 \mu \mathrm{M}$ cisplatin were used as the positive control for the ELISA. The protocol was the same for H69, H82 and DMS 114 cells. The experiment was carried out in duplicate and performed two independent times.

\section{Antitumor studies in nude mice}

Twenty 4-week-old male nude mice were obtained from Charles River Laboratories (Wilmington, MA, USA) and acclimatized for one week. They were housed in autoclaved cages with ad libitum access to food and water in HEPA-filtered racks and closely monitored by animal facility staff. DMS 53 cells were harvested and re-suspended in a 1:1 (v/v) solution of serum-free Waymouth's medium and Matrigel matrix (BD Biosciences, San Jose, CA, USA). Two million cells in $100 \mu \mathrm{L}$ were injected subcutaneously between the scapulae of each mouse $(20,21)$. After the tumors reached $100 \mathrm{~mm}^{3}$, the mice were randomized into two groups. The control group $(\mathrm{N}=10)$ was fed AIN76A diet with $10 \%$ lipid level (referred to hereafter as control diet). The treatment group $(\mathrm{N}=10)$ was changed to a diet containing $50 \mathrm{mg}$ capsaicin/ $\mathrm{kg}$ food (which is about $10 \mathrm{mg}$ capsaicin $/ \mathrm{kg}$ body weight of mouse per day) in the AIN76A diet. Mice were weighed once per week. Their food consumption was monitored daily and their water consumption was monitored once a week. The administration of capsaicin caused no discomfort or weight loss in mice. Additionally, food intake was similar between control and capsaicin-treated mice.

Capsaicin treatment was continued until tumors of the control group reached $1000 \mathrm{~mm}^{3}$. Tumor lengths (l), widths (w) and height (h) were measured daily (6 days a week) for each mouse. Tumor volumes were calculated as $(1 \times w \times h) / 2(22)$. After euthanizing the mice, the tumors were excised. Half of the tumor was snap frozen in liquid nitrogen and used to make lysates. Tumor lysates were prepared using T-Per lysis buffer (Pierce Biotechnology, Rockford, IL, USA), according to manufacturer's protocol $(20,23,24)$. The other half of the tumor was fixed in formalin and used for immunohistochemistry. The caspase-3 activity 
assay was performed with tumor lysates prepared from control mice and capsaicin-treated mice using the caspase-3 activity kit (Chemicon, Temecula, CA, USA). The results obtained by the caspase- 3 activity assay kit were confirmed by cell death ELISA and cleaved PARP ELISA.

\section{Immunohistochemistry}

Human SCLC tissue microarray slides containing 70 human SCLC tissue (isolated from patients) with 10 matched normal lung tissue (Array \#LC802a; US Biomax, Rockville, MD, USA) were deparaffinized and rehydrated as described previously (20). The immunostaining was performed using Vectastain ABC Kit (Vector Laboratories, Burlingame, CA, USA) following the manufacturer's protocol. The array was stained with polyclonal TRPV6 antibody (1:25 dilution). The slides were counterstained with hematoxylin, dehydrated, mounted in Permount Mounting Medium (Fisher Biotech) and photographed by phase contrast microscopy (Leica Microsystems, Wetzlar, Germany) at a magnification of 400X. The expression of TRPV6 was quantitated by using the Leica Application Suite Image Analysis software Version 3.4.1.

\section{Transient receptor potential vanilloid (TRPV) receptor ELISA assays}

The expression of TRPV6 receptors in human SCLC cell lines and normal lung cells was analyzed by using ELISA Kits (Antibodies Online Inc., Atlanta, GA, USA) according to manufacturer's protocols. Cell lysates were prepared according to instructions provided by the kit. The protein concentration of the lysates was measured by the Bradford Assay. An aliquot of the cell lysate containing one hundred micrograms of protein was used to perform the ELISA. The absorbance obtained in NHBEs was taken to be 1 and the relative expression of TRPV6 in the other normal lung cells and human SCLCs were calculated as fold-increase over NHBEs. Each of these assays was completed in duplicate, and the whole experiment was performed two independent times for each cell line.

\section{siRNA transfection and assays}

The siRNA-transfection experiments were performed in DMS 53 and DMS 114 human SCLC cells $(20,24)$. TRPV6 siRNA or control-siRNA (Santa Cruz Biotechnology) were transfected in DMS 53 cells (at 50\% confluence) at a concentration of $75 \mathrm{nM}$ using Oligofectamine reagent (Invitrogen, Carlsbad, CA, USA), according to the manufacturer's protocol $(20,24)$. Eighteen hours post-transfection, the cells were incubated in Waymouth's media containing $1 \%$ FBS and treated with $50 \mu \mathrm{M}$ capsaicin for 36 hours. The effect of TRPV6 siRNA on the apoptotic activity of capsaicin was analyzed by the caspase-3 activity kit (Millipore). The results of the caspase-3 activity assay were confirmed by cleaved PARP ELISA. A non-targeting siRNA sequence was used as the control-siRNA for the transfection experiments $(12,20,24)$. The entire experiment was repeated with a second TRPV6 siRNA (Ambion, Grand Island, NY, USA). Each transfection was performed in duplicate, and the entire assay was performed two independent times. The protocol for transfection was identical in DMS 114 cells.

Western blotting experiments were performed to examine the expression of the TRPV6 after siRNA transfection in all the SCLC cell lines (12). GAPDH was used as the loading control 
and the results of the western blotting assays were quantitated by ImageJ $1.46 \mathrm{p}$. The efficiency of TRPV6 siRNA transfection was also verified by the TRPV6 ELISA kit.

\section{Measurement of calpain activity}

DMS 53 or DMS 114 human SCLC cells were treated with $50 \mu \mathrm{M}$ capsaicin (for 36 hours)

in Waymouth's medium containing 1\% FBS. Subsequently, cells were harvested and washed twice with PBS. Cell lysates were prepared using the assay buffer provided in the Sensolyte 520 Calpain Activity Assay Kit. An aliquot of the cell lysate containing two hundred micrograms of protein was for each reaction. The samples were incubated with $50 \mu \mathrm{l}$ of calpain substrate for 60 minutes at $37^{\circ} \mathrm{C}(25)$. The rest of the assay was performed according to manufacturer's instructions. The fluorescence intensity was measured using a Biotek Synergy2 spectrofluorometer (Biotek Instruments, Winooski, VT, USA) at excitation and emission wavelengths of 490 and $520 \mathrm{~nm}$, respectively. Each sample was measured in duplicate and the whole experiment was performed two independent times. Calpain activity in untreated lysates was considered to be equal to 1 , and the activity observed in treated lysates was calculated as fold increase relative to the untreated control sample.

Tumor lysates were prepared using T-Per lysis buffer (Pierce Biotechnology), according to manufacturer's protocol $(20,24)$. Lysate containing $200 \mu \mathrm{g}$ of protein was used for each calpain assay reaction.

\section{Statistical analysis}

All data was plotted using GraphPad Prism 5 Software, Inc (La Jolla, CA, USA), and results were represented as the mean \pm standard error of the mean (SEM). Results from the control and treated samples were compared using an analysis of variance (ANOVA) followed by a Neumann-Keuls multiple comparison test. All analyses were completed using a 95\% confidence interval. Data was considered significant when $\mathrm{p}<0.05$.

\section{Results}

\section{Capsaicin causes greater apoptosis in human SCLC cell lines than in normal lung cells}

Caspase-3 activity assays revealed that capsaicin caused programmed cell death in H69 human SCLC cells at 36 hours. Capsaicin-induced apoptosis was observed to be maximal at $50 \mu \mathrm{M}$ and remained relatively constant thereafter (Fig. 1A). Next, we measured the timekinetics of capsaicin-induced apoptosis in $\mathrm{H} 69$ cells. We found that capsaicin caused increased cell death at 24 hours but apoptosis was maximal at approximately 36 hours (Fig. 1B). Therefore, we used $50 \mu \mathrm{M}$ capsaicin and a time point of 36 hours for all our experiments. The apoptotic activity of capsaicin was also observed in three additional human SCLC cell lines, DMS 114, DMS 53 and H82 (Fig. 1C). The results obtained in the caspase-3 activity assay were verified by the TUNEL assay, and similar results were obtained (Supplementary Figure 1a). The pro-apoptotic activity of capsaicin was also measured by a Cell Death ELISA kit (Supplementary Fig. 1c) and a PARP Cleavage ELISA kit (Supplementary Fig. 1e). The results obtained by all the four apoptosis assays were similar. 
Next, we wanted to examine the apoptotic activity of capsaicin in normal lung cells. We observed that capsaicin demonstrated meagre to no apoptotic activity in normal human small airway epithelial cells (SAEC) and normal human bronchial epithelial cells (NHBE) (Fig. 1D). These results were confirmed using a TUNEL assay, and similar results were obtained (Supplementary Fig. 1b). We also measured the apoptotic activity of capsaicin in normal lung cells using a Cell Death ELISA kit (Supplementary Fig. 1d) and the PARP Cleavage ELISA kit (Supplementary Fig. 1f). Our results from all four apoptosis assays showed that capsaicin-induced apoptosis was specific for SCLC cells but did not affect normal lung cells.

\section{Capsaicin suppressed the growth of DMS 53 human SCLC in vivo}

The anti-neoplastic activity of capsaicin was measured in vivo using a nude mouse model $(12,20)$. DMS 53 human SCLC cells were injected between the scapulae of nude mice. The tumors were allowed to grow until approximately $100 \mathrm{~mm}^{3}$, after which they were randomized into two groups of ten mice each. The control group was administered AIN-76A diet with 10\% lipid level (control diet). The treatment group was administered $50 \mathrm{mg}$ capsaicin $/ \mathrm{kg}$ food. We observed that that the dietary administration of capsaicin decreased the tumor growth rate of DMS 53 human SCLC tumors xenografts in nude mice (Fig. 2A). The administration of $50 \mathrm{mg}$ capsaicin $/ \mathrm{kg}$ food in the diet was well tolerated and caused no apparent discomfort or weight loss in mice. Additionally, food intake and water consumption was similar between both groups (Supplementary Fig. 2a). Caspase-3 activity assays indicate that DMS 53 tumor lysates from capsaicin-treated mice displayed about 2-3 fold greater apoptosis relative to control mice (Fig. 2B). These results were confirmed by the Cell Death ELISA (Supplementary Fig.2b) and Cleaved PARP ELISA experiments and similar results were obtained (Supplementary Fig. 2c).

Hemotoxylin and Eosin ( $\mathrm{H}$ and $\mathrm{E})$ staining of the tumors revealed the presence of apoptotic bodies in capsaicin-treated DMS 53 tumors (Fig. 2C) isolated from nude mice. HPLC analysis (26) indicates that capsaicin was detected in the SCLC tumors of athymic mice fed with capsaicin-containing food. No capsaicin was found in the tumors of mice fed with control AIN-76A diet (Fig. 2D).

\section{Capsaicin induces apoptosis in human SCLC cells via TRPV6}

The subsequent experiments aimed to investigate the molecular mechanisms underlying the apoptotic activity of capsaicin. The biological activity of capsaicin is mediated by TRPV receptors (27). The treatment of DMS 53 and DMS 114 human SCLC cells with Ruthenium Red, a generalized TRPV antagonist (16), abrogated the apoptotic activity of capsaicin, indicating that TRPV receptors are involved in the pro-apoptotic effects of apoptosis (Supplementary Fig. 3a). Capsaicin is a ligand for the TRPV1 receptor. We wanted to examine the role of TRPV1 in capsaicin-induced apoptosis. Specific chemical inhibitors of TRPV1, namely capsazepine and SB366791, did not affect the apoptotic activity of capsaicin in DMS 53 and DMS 114 human SCLC cells (Supplementary Fig. 3b-c). These results suggested that the apoptotic activity of capsaicin did not require TRPV1. We confirmed these results with a second apoptosis assay namely the TUNEL assay and analogous results were obtained (data not shown). 
Out of the entire TRPV receptor family, capsaicin only regulates TRPV1 and TRPV6 (13). Therefore, we conjectured that TRPV6 may be responsible for the apoptotic effects of capsaicin. Studies performed in other experimental systems have revealed that capsaicin can regulate the bioactivity of the TRPV6. Therefore, we decided to examine whether capsaicininduced apoptosis was mediated by TRPV6. As a first step we investigated whether human SCLC cell lines expressed TRPV6. Western blotting showed that TRPV6 was robustly expressed by the four human SCLC cell lines used in the study (Fig. 3A). These results were confirmed using a TRPV6 ELISA kit (Antibodies Online), and the results agreed with those obtained in the immunoblotting experiments (Fig. 3B). We observed that the normal lung epithelial cells, namely NHBE and SAEC, expressed lower levels of TRPV6 than human SCLC cell lines. These data may, in part, explain the relative selectivity of capsaicin for human SCLC cells over normal lung cells. We wanted to investigate whether such differential expression of TRPV6 also existed in SCLC tissues from patients. We immunostained a TMA slide containing 70 human SCLC tissue with 10 matched normal lung tissues. We observed strong cytoplasmic staining for TRPV6 in the human SCLC tissues (Fig. 3D), whereas very little staining was observed in the matched normal tissues (Fig. 3C). We quantitated the expression of TRPV6 using image analysis. The level of TRPV6 found in human SCLC tissues was 4-5 fold higher than normal lung epithelial tissue (Fig. 3E).

The role of TRPV6 in capsaicin-induced apoptosis in human SCLC cell lines was examined by siRNA methodology. Caspase-3 activity assays revealed that the transfection of TRPV6 siRNA (referred to as TRPV6 siRNA 1) reversed the apoptotic activity of capsaicin in DMS 53 and DMS 114 human SCLC cells. The siRNA transfection experiments were repeated using a second independent TRPV6 siRNA (referred to as TRPV6 siRNA 2 obtained from Ambion, Inc.) and similar results were observed (Fig. 4A). The transfection of a control non-targeting siRNA did not have any impact on capsaicin-induced apoptosis in human SCLC cells (Fig. 4A, third set of bars from the left). Furthermore, both sets of TRPV6 siRNA do not induce any apoptosis in untreated DMS 53 and DMS 114 cells (Fig. 4B). Similarly, cleaved PARP ELISA assays revealed that the transfection of TRPV6 siRNA ablated the apoptotic effects of capsaicin (Fig. 4C). Cleaved PARP ELISA assays also reveal that both TRPV6 siRNA 1 and TRPV6 siRNA 2 did not cause apoptosis in untreated DMS 53 and DMS 114 human SCLC cells (Fig. 4D).

The effects of both TRPV6 siRNAs on capsaicin-induced apoptosis were also confirmed by the TUNEL assay (Supplementary Fig. 4a and b) and analogous results were obtained. Western blotting experiments and TRPV6-ELISA assays confirmed that both the sets of TRPV6 siRNA suppressed the expression of TRPV6 in DMS 53 and DMS 114 human SCLC cells (Supplementary Fig. 5a and b).

\section{Capsaicin causes calcium-dependent activation of calpain-1 and calpain-2 activity}

The role of calcium homeostasis in capsaicin-induced apoptosis was analyzed using the intracellular calcium chelator BAPTA-AM. Figure 5A shows that 10 $\mu \mathrm{M}$ BAPTA-AM significantly attenuated capsaicin-induced apoptosis in DMS 53 and DMS 114 human SCLC cells, as measured by the caspase- 3 activity assay. The inhibitory effects of BAPTA-AM 
were also confirmed by the TUNEL assay and similar results were obtained (Supplementary Fig. 5a). This suggests that capsaicin is causing apoptosis by elevation of intracellular calcium. In addition, both caspase-3 activity assays (Fig. 5B) and TUNEL assays (Supplementary Fig. 5b) revealed that the apoptotic activity of capsaicin was significantly suppressed by the calpain inhibitor calpeptin indicating that the activation of calpains 1 and 2 may be mediating the apoptotic effects of capsaicin.

The effect of capsaicin on total calpain activity was examined in DMS 53 human SCLC cells. We observed that capsaicin increased calpain activity by approximately 3 -fold in DMS 53 and DMS 114 human SCLC cells (Fig. 5C). Capsaicin-induced calpain activity was reversed by the calcium chelator BAPTA-AM (Fig. 5C). Capsaicin did not have any effect on the expression levels of calpain 1 or calpain 2 (data not shown). Next we analyzed the relative calpain activity of tumors obtained from the nude mice experiments. We observed that tumors isolated from capsaicin-fed mice displayed higher calpain activity than tumors isolated from control-diet fed mice (Fig. 5D).

An important question is whether capsaicin induces calpain activity via TRPV6 receptors. The transfection of TRPV6 siRNA (referred to as TRPV6 siRNA 1) suppressed capsaicininduced calpain activation in DMS 53 cells (Fig. 6A). The siRNA transfection experiments were repeated using a second independent TRPV6 siRNA (referred to TRPV6 siRNA 2, Ambion Inc.) and similar results were obtained. In contrast, capsaicin-induced calpain activity was unaffected by the non-targeting control-siRNA. Similarly both sets of TRPV6 siRNA did not have any influence on the basal calpain activity of untreated DMS 53 and DMS 114 cells (Fig. 6B). Western Blotting and TRPV6-ELISA experiments revealed that both the TRPV6 siRNA caused a robust decrease in TRPV6 expression in DMS 114 and DMS 53 cells (Supplementary Fig. 6a and 6b). Taken together, capsaicin induced apoptosis in human SCLC cells via TRPV6 receptors and downstream activation of calpain family of proteins.

\section{Discussion}

The TRPV receptor family is comprised of six members, TRPV1-6 $(28,29)$. The apoptotic activity of capsaicin was independent of the TRPV1 receptor and mediated by the TRPV6 receptor in human SCLCs. When we observed that the pro-apoptotic activity of capsaicin is independent of TRPV1 (however mediated by the TRPV family), we began to look for other TRPV proteins which could play a role in this process. We chose TRPV6 for several reasons. Previous studies indicate that the TRPV6 receptor is directly involved in the survival of human prostate cancer cells (28). Experiments in transfected cells, gastric cancer and prostate cancer cells have revealed TRPV6 to be one of the direct cellular targets of capsaicin activity $(13,28)$. In addition, capsaicin was found to cause apoptosis in human gastric cancer cells via the TRPV6 pathway(13). We conjectured that perhaps TRPV6 was involved in capsaicin-induced apoptosis of human SCLC cells.

The TRPV6 is one of the most calcium selective ion channel in the TRPV family. Its main function is to regulate calcium transport, reabsorption and homeostasis in epithelial tissues $(30,31)$. Recent studies have shown that the TRPV6 receptor is also over-expressed in 
carcinomas of ovary, breast, colon, prostate and thyroid (28, 32-34). Our results show that the expression of TRPV6 in normal lung cells was substantially lower than in human SCLC cell lines and tissues from patients. Such differential expression of TRPV6 between normal and neoplastic cells could form the basis of novel therapeutic interventions in human SCLCs.

Although, the TRPV6 is a ligand-independent cation channel, its expression and function are tightly regulated by diverse signaling proteins such as G-protein coupled receptors, androgen receptor, calmodulin, tyrosine kinases like Src and protein phosphastases like PP2 $(30,31,35)$. In addition, several nutritional compounds have been shown to influence the activity of TRPV6. These include curcumin, vitamin D, beta-ionone, fatty acids and capsaicin $(28,36)$. Capsaicin increases TRPV6 expression in human gastric and prostate cancer cells, and this could potentially lead to excessive increase of intracellular calcium $(13,15,34,37)$ and trigger apoptosis. In contrast, in Cui et al., (2002) high concentrations of capsaicin $(250 \mu \mathrm{M})$ blocked the influx of calcium ions in TRPV6-transfected Jurkat cells (38). Such differences may be ascribed to the fact that the studies of Cui et al., (2002) were performed on overexpressed TRPV6, whereas the studies involving gastric cancer cells were performed on endogenous TRPV6. Recent observations by Spehr et al., (2011) have shown that overexpression of TRPV6 leads to constitutively active calcium-channels whereas endogenous TRPV6 is not constitutively active (35). Our results provide yet another scenario where capsaicin regulates the endogenous TRPV6 pathway to induce apoptosis in human SCLCs. An interesting question emerges from these results; how is capsaicin contacting the TRPV6 receptors in human cancer cells? Very little is known about the effect of dietary components (like capsaicin) on upstream signaling events involving the TRPV6 receptor. Our future experiments will endeavor to delineate the upstream biochemical mechanisms by which capsaicin regulates the TRPV6 receptor in normal and malignant cells.

The apoptotic activity of capsaicin was found to be dependent on intracellular calcium and the calpain pathway $(7,15)$. The calpains are a family of calcium-dependent intracellular cysteine proteases, which regulate multiple cellular processes like cell division, differentiation, migration and apoptosis (39). Nutritional compounds like flavonoids and tea polyphenols have been shown to activate the calpain pathway to execute apoptosis in human cancer cells $(25,40,41),(7,15)$. Our data shows for the first time that capsaicin-induced activation of calpain activity occurs downstream of the TRPV6 receptor in human SCLCs. The TRPV1 receptor is known to cause calpain activation (42-44), however no link has been reported between TRPV6 and the calpain pathway. Taken together, our results suggest that dietary compounds (or small molecules) target the TRPV6 receptor may be useful for the management and therapy of human SCLC.

\section{Supplementary Material}

Refer to Web version on PubMed Central for supplementary material. 


\section{Acknowledgments}

We thank Dr. Srikumar Chellappan and his laboratory for their help and support. This work was supported by the grants Young Clinical Scientist Award (\#82115) from the Flight Attendant Medical Association, Miami, FL, the NIH R15 AREA grant (1R15CA161491-01A1) and a AICR research grant to PDG. RGC was the recipient of a summer research fellowship is supported by grants from the West Virginia IDeA Network of Biomedical Research Excellence (P20RR016477 and P20GM103434).

\section{References}

1. Kallianos A, Rapti A, Zarogoulidis P, et al. Therapeutic procedure in small cell lung cancer. Journal of thoracic disease. 2013; 5:S420-S424. [PubMed: 24102016]

2. Goldberg SB, Willers H, Heist RS. Multidisciplinary management of small cell lung cancer. Surg Oncol Clin N Am. 2013; 22:329-343. [PubMed: 23453338]

3. Aggarwal BB, Kunnumakkara AB, Harikumar KB, Tharakan ST, Sung B, Anand P. Potential of spice-derived phytochemicals for cancer prevention. Planta Med. 2008; 74:1560-1569. [PubMed: 18612945]

4. Lee JH, Khor TO, Shu L, Su ZY, Fuentes F, Kong AN. Dietary phytochemicals and cancer prevention: Nrf2 signaling, epigenetics, and cell death mechanisms in blocking cancer initiation and progression. Pharmacol Ther. 2013; 137:153-171. [PubMed: 23041058]

5. Wang H, Khor TO, Shu L, et al. Plants vs. cancer: a review on natural phytochemicals in preventing and treating cancers and their druggability. Anti-cancer agents in medicinal chemistry. 2012; 12:1281-1305. [PubMed: 22583408]

6. Reyes-Escogido Mde L, Gonzalez-Mondragon EG, Vazquez-Tzompantzi E. Chemical and pharmacological aspects of capsaicin. Molecules. 2011; 16:1253-1270. [PubMed: 21278678]

7. Lau, JK.; Brown, KC.; Dom, AM.; Dasgupta, P. Capsaicin: Potential Applications in Cancer Therapy. Bentham Press Inc.; London, United Kingdom: 2012.

8. Anandakumar P, Kamaraj S, Jagan S, Ramakrishnan G, Vinodhkumar R, Devaki T. Stabilization of pulmonary mitochondrial enzyme system by capsaicin during benzo(a)pyrene induced experimental lung cancer. Biomed Pharmacother. 2007

9. Park KK, Surh YJ. Effects of capsaicin on chemically-induced two-stage mouse skin carcinogenesis. Cancer Lett. 1997; 114:183-184. [PubMed: 9103286]

10. Tanaka T, Kohno H, Sakata K, et al. Modifying effects of dietary capsaicin and rotenone on 4nitroquinoline 1-oxide-induced rat tongue carcinogenesis. Carcinogenesis. 2002; 23:1361-1367. [PubMed: 12151355]

11. Zhang Z, Huynh H, Teel RW. Effects of orally administered capsaicin, the principal component of capsicum fruits, on the in vitro metabolism of the tobacco-specific nitrosamine NNK in hamster lung and liver microsomes. Anticancer Res. 1997; 17:1093-1098. [PubMed: 9137455]

12. Brown KC, Witte TR, Hardman WE, et al. Capsaicin displays anti-proliferative activity against human small cell lung cancer in cell culture and nude mice models via the E2F pathway. PLoS One. 2010; 5:e10243. [PubMed: 20421925]

13. Chow J, Norng M, Zhang J, Chai J. TRPV6 mediates capsaicin-induced apoptosis in gastric cancer cells--Mechanisms behind a possible new "hot" cancer treatment. Biochim Biophys Acta. 2007; 1773:565-576. [PubMed: 17292493]

14. Thoennissen NH, O'Kelly J, Lu D, et al. Capsaicin causes cell-cycle arrest and apoptosis in ERpositive and -negative breast cancer cells by modulating the EGFR/HER-2 pathway. Oncogene. 2010; 29:285-296. [PubMed: 19855437]

15. Ziglioli F, Frattini A, Maestroni U, Dinale F, Ciufifeda M, Cortellini P. Vanilloid-mediated apoptosis in prostate cancer cells through a TRPV-1 dependent and a TRPV-1-independent mechanism. Acta Biomed. 2009; 80:13-20. [PubMed: 19705615]

16. Clapham DE, Julius D, Montell C, Schultz G. International Union of Pharmacology. XLIX. Nomenclature and structure-function relationships of transient receptor potential channels. Pharmacol Rev. 2005; 57:427-450. [PubMed: 16382100] 
17. Amantini C, Ballarini P, Caprodossi S, et al. Triggering of transient receptor potential vanilloid type 1 (TRPV1) by capsaicin induces Fas/CD95-mediated apoptosis of urothelial cancer cells in an ATM-dependent manner. Carcinogenesis. 2009; 30:1320-1329. [PubMed: 19502594]

18. Amantini C, Mosca M, Nabissi M, et al. Capsaicin-induced apoptosis of glioma cells is mediated by TRPV1 vanilloid receptor and requires p38 MAPK activation. J Neurochem. 2007; 102:977990. [PubMed: 17442041]

19. Gil YG, Kang MK. Capsaicin induces apoptosis and terminal differentiation in human glioma A172 cells. Life Sci. 2008; 82:997-1003. [PubMed: 18405923]

20. Lau JK, Brown KC, Thornhill BA, et al. Inhibition of cholinergic signaling causes apoptosis in human bronchioalveolar carcinoma. Cancer Res. 2013; 73:1328-1339. [PubMed: 23222296]

21. Song P, Sekhon HS, Fu XW, et al. Activated cholinergic signaling provides a target in squamous cell lung carcinoma. Cancer Res. 2008; 68:4693-4700. [PubMed: 18559515]

22. Shabbir M, Thompson CS, Mikhailidis D, Morgan RJ, Burnstock G. Extracellular ATP attenuates the growth of hormone refractory prostate cancer in vivo. Eur Urology Supplements. 2003; 2:2427.

23. Kinkade R, Dasgupta P, Carie A, et al. A small molecule disruptor of Rb/Raf-1 interaction inhibits cell proliferation, angiogenesis, and growth of human tumor xenografts in nude mice. Cancer Res. 2008; 68:3810-3818. [PubMed: 18483265]

24. Brown KC, Perry HE, Lau JK, et al. Nicotine Induces the Up-regulation of the alpha7-Nicotinic Receptor (alpha7-nAChR) in Human Squamous Cell Lung Cancer Cells via the Sp1/GATA Protein Pathway. J Biol Chem. 2013; 288:33049-33059. [PubMed: 24089524]

25. Guha P, Dey A, Dhyani MV, et al. Calpain and caspase orchestrated death signal to accomplish apoptosis induced by resveratrol and its novel analog hydroxystilbene-1 [correction of hydroxstilbene-1] in cancer cells. J Pharmacol Exp Ther. 2010; 334:381-394. [PubMed: 20484155]

26. Kawada T, Watanabe T, Katsura K, Takami H, Iwai K. Formation and metabolism of pungent principle of Capsicum fruits. XV. Microdetermination of capsaicin by high-performance liquid chromatography with electrochemical detection. Journal of chromatography. 1985; 329:99-105. [PubMed: 4019637]

27. Santoni G, Farfariello V. TRP channels and cancer: new targets for diagnosis and chemotherapy. Endocrine, metabolic \& immune disorders drug targets. 2011; 11:54-67.

28. Lehen'kyi V, Raphael M, Prevarskaya N. The role of the TRPV6 channel in cancer. The Journal of physiology. 2012; 590:1369-1376. [PubMed: 22331416]

29. Gunthorpe MJ, Szallasi A. Peripheral TRPV1 receptors as targets for drug development: new molecules and mechanisms. Curr Pharm Des. 2008; 14:32-41. [PubMed: 18220816]

30. Hoenderop JG, Bindels RJ. Calciotropic and magnesiotropic TRP channels. Physiology. 2008; 23:32-40. [PubMed: 18268363]

31. Billeter AT, Hellmann JL, Bhatnagar A, Polk HC Jr. Transient receptor potential ion channels: powerful regulators of cell function. Annals of surgery. 2014; 259:229-235. [PubMed: 23989052]

32. Bowen CV, DeBay D, Ewart HS, et al. In vivo detection of human rich tumors with anti-cancer peptides derived from soricidin. PLoS One. 2013; 8:e58866. [PubMed: 23554944]

33. Bolanz KA, Hediger MA, Landowski CP. The role of TRPV6 in breast carcinogenesis. Molecular cancer therapeutics. 2008; 7:271-279. [PubMed: 18245667]

34. Wissenbach U, Niemeyer B, Himmerkus N, Fixemer T, Bonkhoff H, Flockerzi V. TRPV6 and prostate cancer: cancer growth beyond the prostate correlates with increased TRPV6 Ca2+ channel expression. Biochem Biophys Res Commun. 2004; 322:1359-1363. [PubMed: 15336984]

35. Spehr J, Gelis L, Osterloh M, et al. G protein-coupled receptor signaling via Src kinase induces endogenous human transient receptor potential vanilloid type 6 (TRPV6) channel activation. The Journal of biological chemistry. 2011; 286:13184-13192. [PubMed: 21349844]

36. Fukushima A, Aizaki Y, Sakuma K. Short-chain fatty acids induce intestinal transient receptor potential vanilloid type 6 expression in rats and Caco-2 cells. The Journal of nutrition. 2009; 139:20-25. [PubMed: 19056662] 
37. Venier N, Colquhoun A, Loblaw A, Fleshner N, Klotz L, Venkateswaran V. 1189 capsaicin reduces proliferation and induces apoptosis in prostate cancer cells via TRPV6 mediated phenomenon. The Journal of Urology. 2010; 183:e460-e461.

38. Cui J, Bian JS, Kagan A, McDonald TV. CaT1 contributes to the stores-operated calcium current in Jurkat T-lymphocytes. The Journal of biological chemistry. 2002; 277:47175-47183. [PubMed: 12361955]

39. Momeni HR. Role of calpain in apoptosis. Cell journal. 2011; 13:65-72. [PubMed: 23507938]

40. Gil-Parrado S, Fernandez-Montalvan A, Assfalg-Machleidt I, et al. Ionomycin-activated calpain triggers apoptosis. A probable role for Bcl-2 family members. The Journal of biological chemistry. 2002; 277:27217-27226. [PubMed: 12000759]

41. Lee MJ, Kee KH, Suh CH, Lim SC, Oh SH. Capsaicin-induced apoptosis is regulated by endoplasmic reticulum stress- and calpain-mediated mitochondrial cell death pathways. Toxicology. 2009; 264:205-214. [PubMed: 19699254]

42. Hong S, Agresta L, Guo C, Wiley JW. The TRPV1 receptor is associated with preferential stress in large dorsal root ganglion neurons in early diabetic sensory neuropathy. J Neurochem. 2008; 105:1212-1222. [PubMed: 18182051]

43. Jaggi AS, Singh N. Mechanisms in cancer-chemotherapeutic drugs-induced peripheral neuropathy. Toxicology. 2012; 291:1-9. [PubMed: 22079234]

44. Rehman R, Bhat YA, Panda L, Mabalirajan U. TRPV1 inhibition attenuates IL-13 mediated asthma features in mice by reducing airway epithelial injury. International immunopharmacology. 2013; 15:597-605. [PubMed: 23453702] 
(A)

(C)

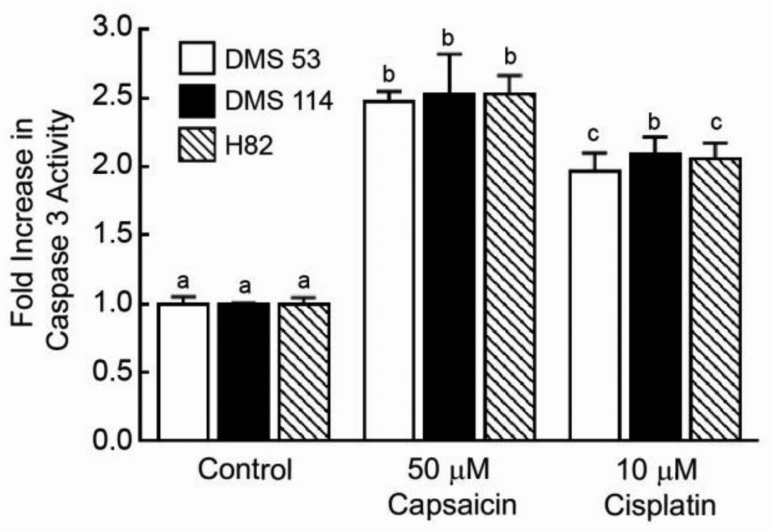

(B)
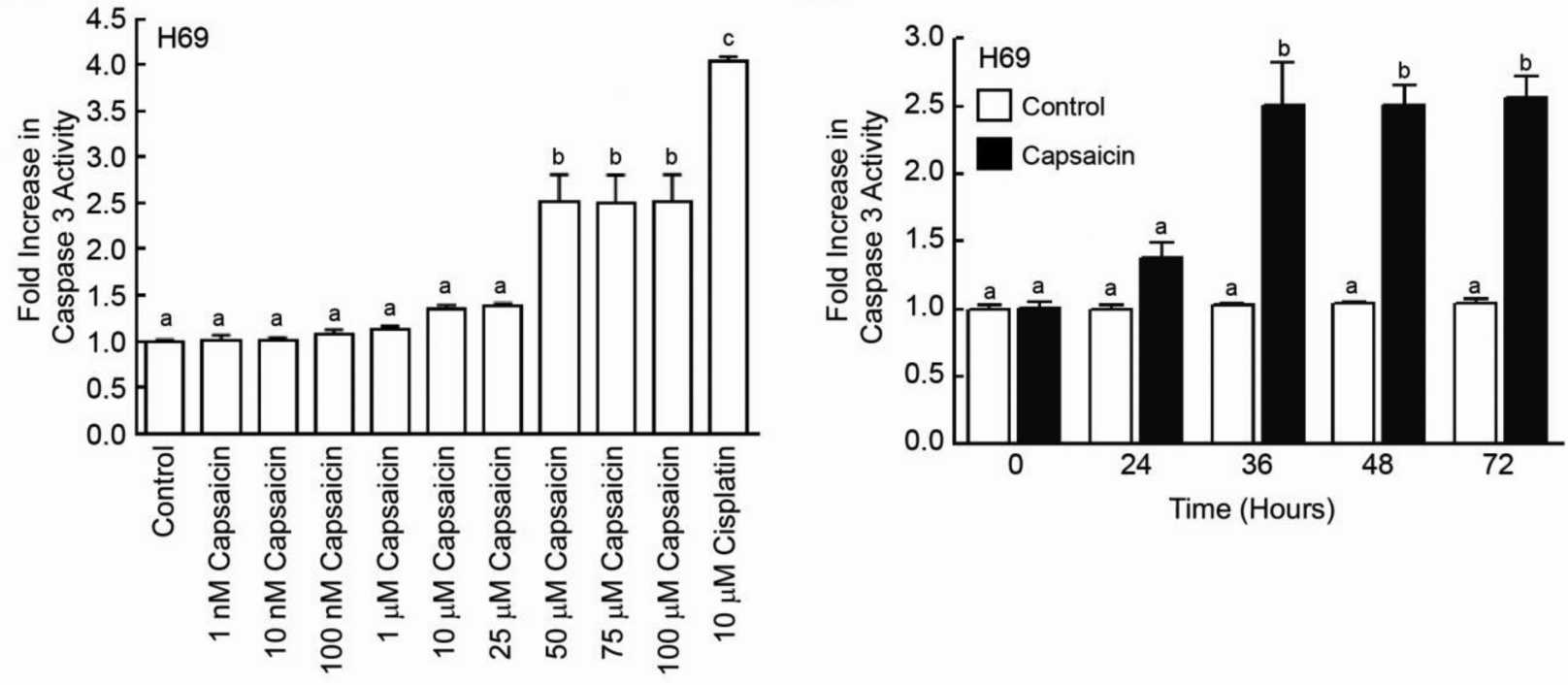

(D)

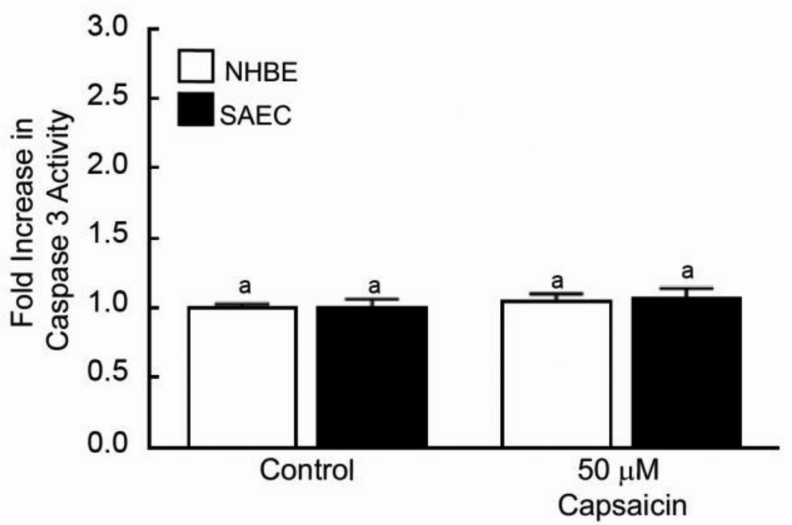

Fig. 1.

Capsaicin induced greater apoptosis in human SCLC cell lines than in normal lung cells. (A) Caspase-3 activity assays reveal that capsaicin caused apoptosis in H69 human SCLC cells. H69 cells treated with $10 \mu \mathrm{M}$ cisplatin for 36 hours were taken as the positive control for the assay. (B) Time-kinetics of capsaicin-induced apoptosis in H69 human SCLC cells. (C) The treatment of DMS 53, DMS 114 and H82 human SCLC cells with $50 \mu \mathrm{M}$ capsaicin caused approximately 2.5 -fold apoptosis in 36 hours, as measured by caspase- 3 activity assay. Cells treated with $10 \mu \mathrm{M}$ cisplatin for 36 hours were used as the positive control for the assay (D) The addition of $50 \mu \mathrm{M}$ capsaicin produced little-to-no apoptosis in normal human bronchial epithelial (NHBE) cells or in normal small airway epithelial cells (SAECs). Each sample was analyzed in duplicate. Caspase-3 activity in untreated lysates was considered to be equal to 1 , and the activity observed in treated lysates was calculated as fold increase relative to the untreated control sample. Data represent mean \pm SEM from two independent experiments. Results indicated by a different letter are significantly different $(P<0.05)$. 
(A)

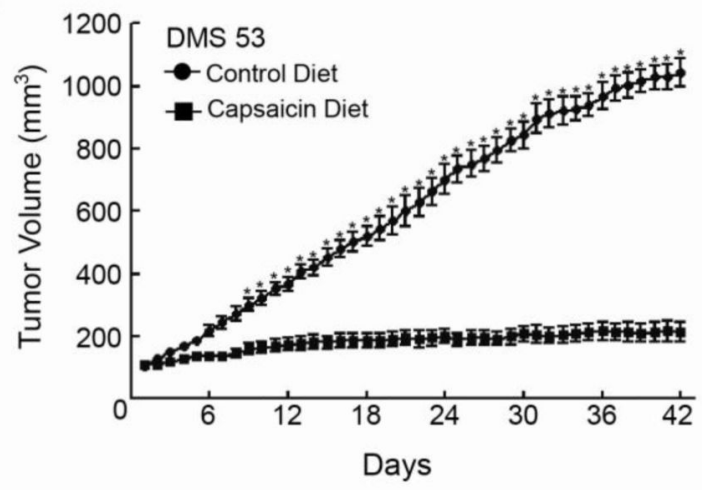

(C)

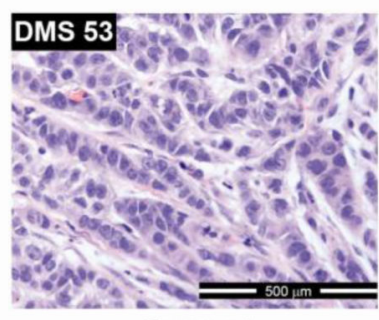

Control

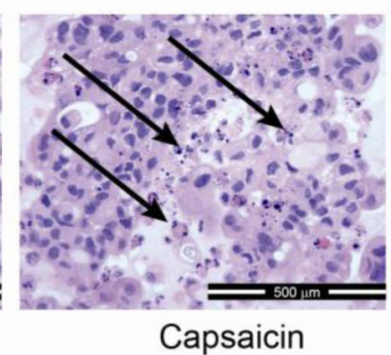

Capsaicin
(B)

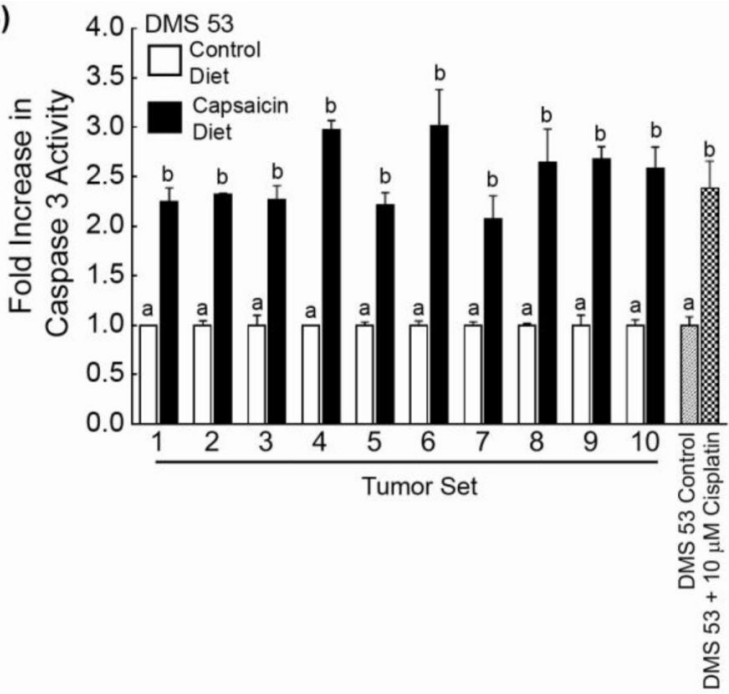

(D)

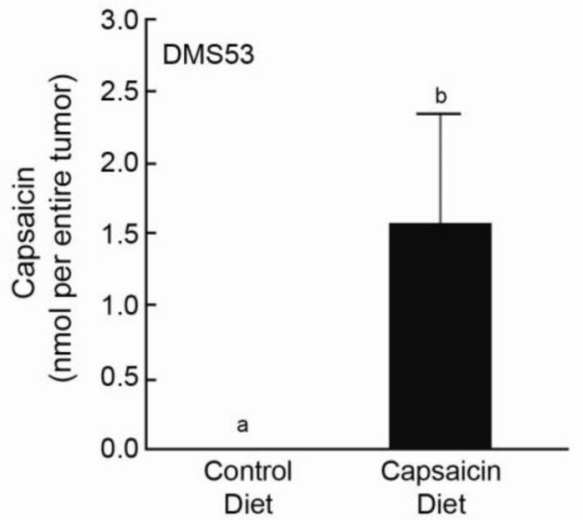

Fig. 2.

Capsaicin induces apoptosis of DMS 53 human SCLC tumors in nude mouse model. (A) The administration of capsaicin ( $50 \mathrm{mg}$ capsaicin $/ \mathrm{kg}$ food) decreased the growth rate of DMS 53 human SCLC tumors xenografted in nude mice ( $\mathrm{n}=10$ mice per group). (B) An aliquot of the DMS 53 tumors from nude mice were frozen and lysates were made.

Caspase- 3 activity of these lysates was measured. The capsaicin-treated DMS 53 tumors had higher caspase-3 activity (indicating higher apoptosis) than the tumors in the control group. Each sample was analyzed in duplicate. The caspase- 3 activity of untreated cells was considered to be equal to 1 , and the caspase- 3 activity of the other samples were as foldincrease relative to the control. Cell lysates made from untreated DMS 53 cells and those treated with $10 \mu \mathrm{M}$ cisplatin for 36 hours were used as the positive control for the assay. (C) Hemotoxylin and Eosin staining show the presence of greater number of apoptotic bodies (indicated by the black arrows) in capsaicin-treated DMS 53 tumors (right panel) relative to tumors treated with vehicle only (left panel). (D) HPLC analysis shows that capsaicin was only detected in the tumor lysates of mice which were fed capsaicin-containing food. Data represent mean \pm SEM. Results indicated by a different letter or an asterisk are significantly different $(P<0.05)$. 
(A)

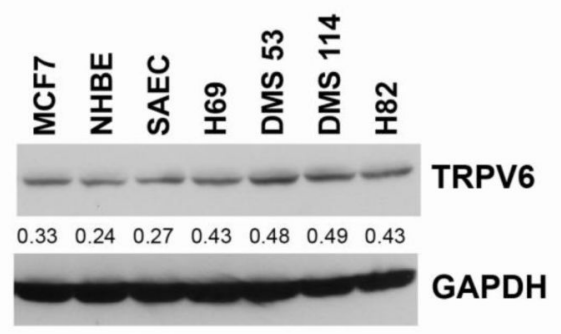

(C)

\section{Normal Human Lung Tissue}
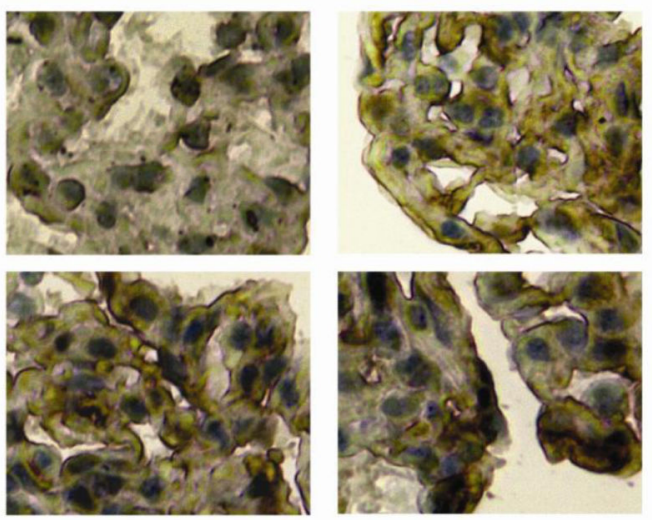

(B)

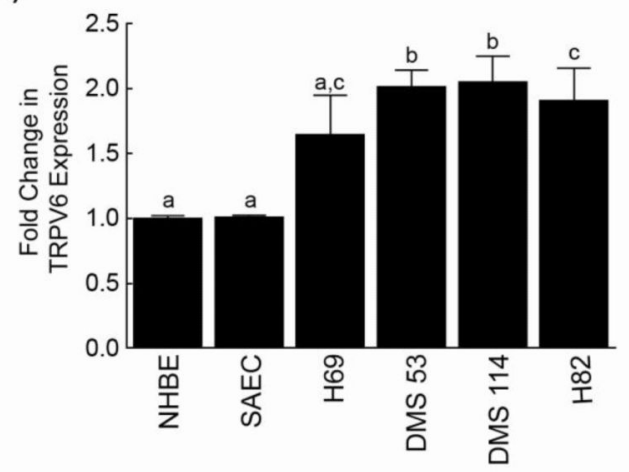

(D)

Human Small Cell Lung Cancer Tissue
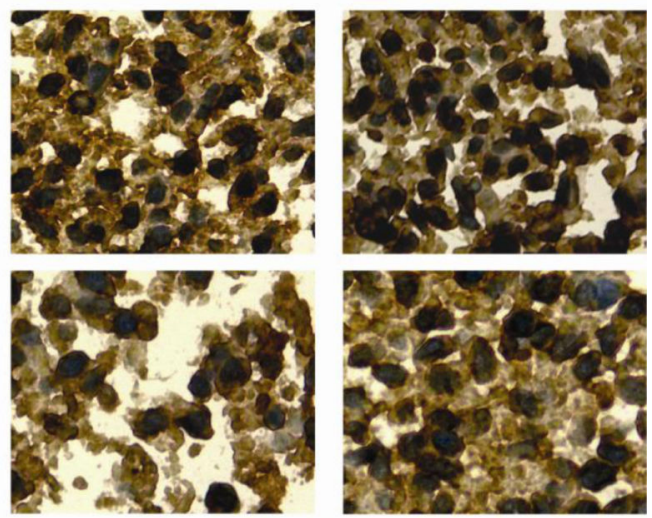

(E)

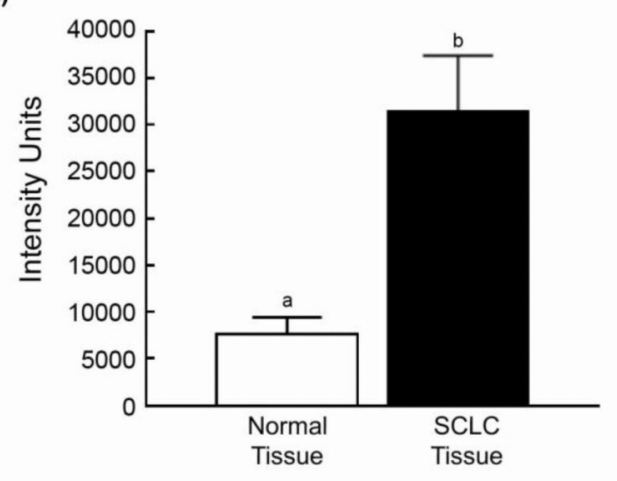

Fig. 3.

Human SCLC cells (and tissues) express greater amounts of TRPV6 than normal lung cells. (A) Immunoblotting experiments show that human SCLC cell lines express TRPV6. MCF-7 cells were used as the positive control in the assay. NHBEs and SAECs express lower levels of TRPV6 than human SCLC cell lines. GAPDH was used as the loading control, and the results were quantitated by densitometric analysis (NIH ImageJ 1.46p). The experiment was carried out two independent times and representative results are shown. (B) ELISA experiments demonstrate that the TRPV6 receptor is expressed in human SCLCs and normal 
lung cells (NHBEs and SAECs). Each sample was measured in duplicate. The entire experiment was performed two independent times. (C) Immunostaining experiments demonstrate that TRPV6 is poorly expressed in normal cells. (D) TRPV6 is robustly expressed in human SCLC tissues (isolated from patients). A tumor microarray (TMA) containing 70 cores of human SCLCs and 10 matched normal lung tissue was used for these experiments and four panels of representative photos are shown. Scale bar $=100 \mu \mathrm{m}$. (E) The levels of TRPV6 expression on the TMA slide were quantitated by image analysis. The level of TRPV6 in human SCLC tumors was found to be substantially higher than normal lung tissue. Data represent mean \pm SEM. Results indicated by a different letter or an asterisk are significantly different $(P<0.05)$. 
(A)

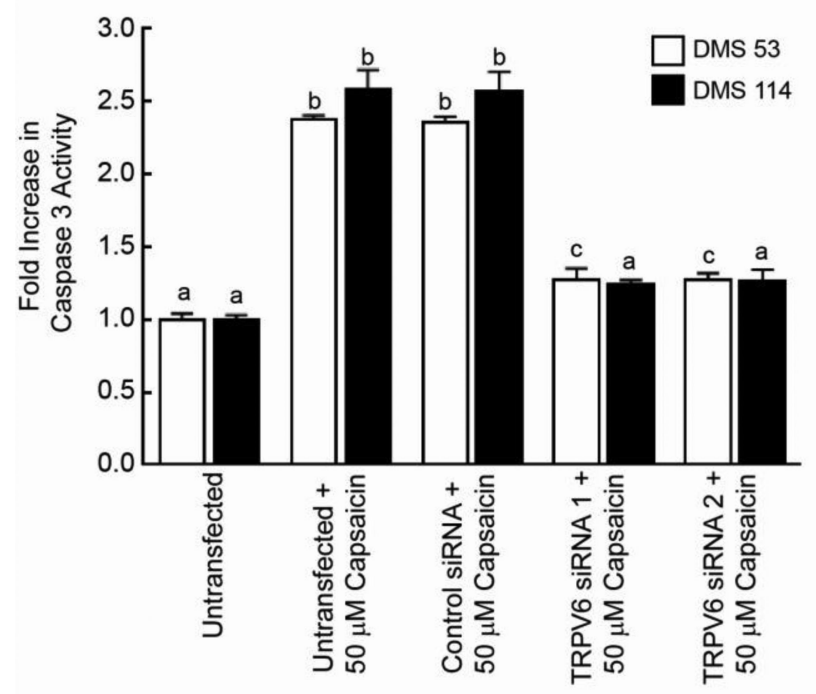

(C)

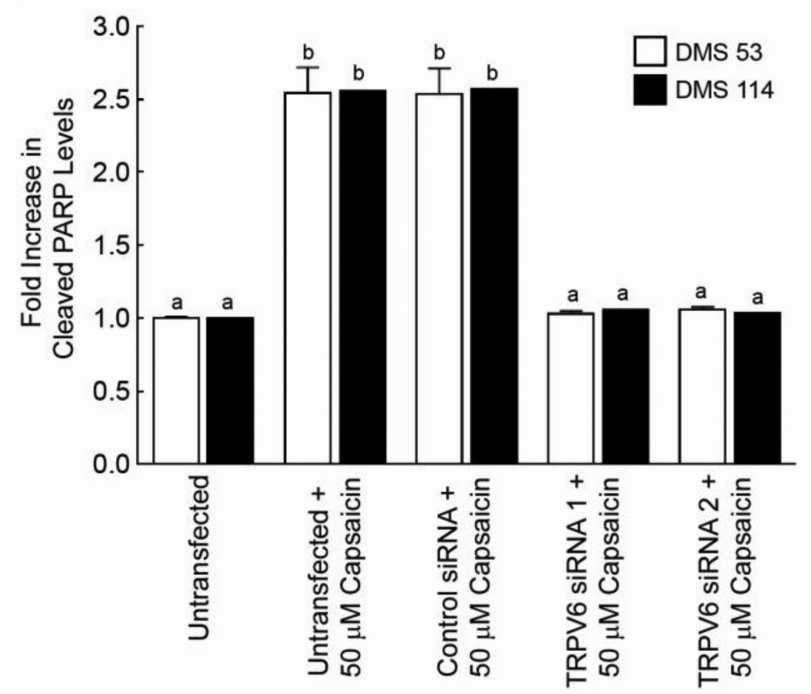

(B)

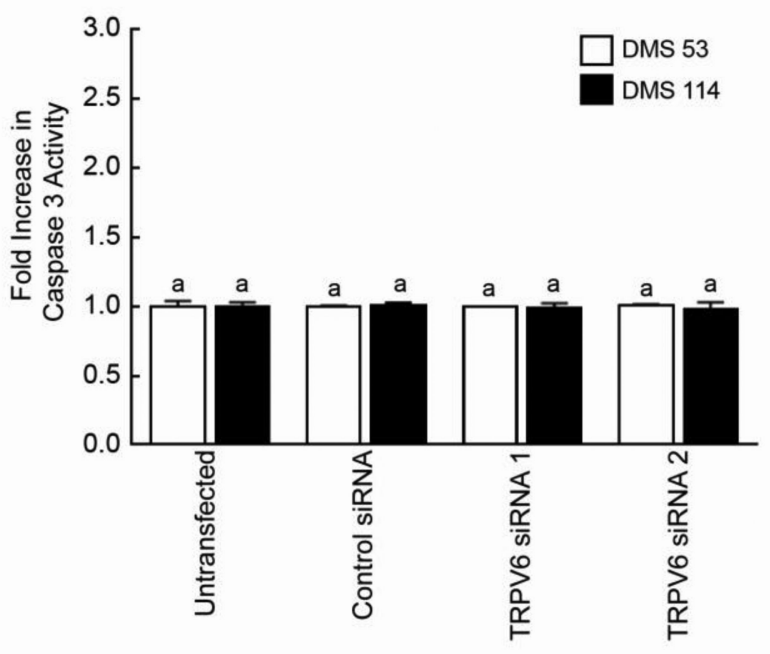

(D)

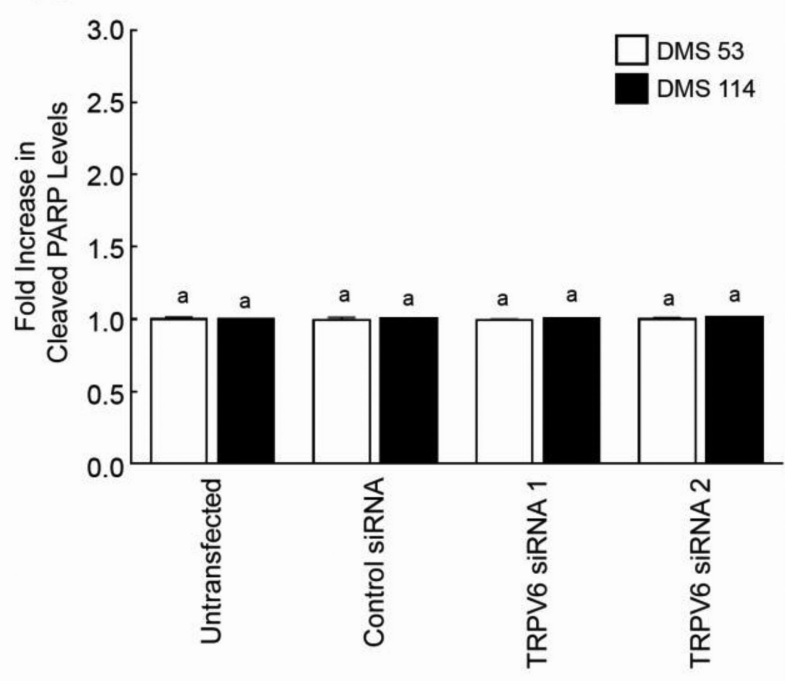

Fig. 4.

Capsaicin-induced apoptosis is mediated by the TRPV6 pathway. (A) Depletion of TRPV6 by siRNA methodology reversed the apoptotic activity of capsaicin in DMS 53 and DMS 114 human SCLCs. TRPV6 siRNA 1 (Santa Cruz Biotechnology) and TRPV6 siRNA 2 (Ambion Inc.) represent two independent TRPV6 siRNA used in the assay. Apoptosis was measured by the caspase- 3 activity assay. No effect on capsaicin-induced apoptosis was observed when the cells were transfected by a non-targeting control-siRNA. (B) The transfection of TRPV6 siRNA did not cause any apoptosis in untreated DMS 53 and DMS 114 human SCLC cells. The caspase-3 activity of untreated cells was considered to be equal to 1 , and the caspase- 3 activity of the other samples were as fold-increase relative to the control. (C) Cleaved PARP ELISA experiments demonstrate that transfection of TRPV6 siRNA 1 ablated the apoptotic effects of capsaicin in human SCLCs. The experiment was repeated with a second independent TRPV6 siRNA (referred to as TRPV6 siRNA 2) and 
similar results were obtained. No effect on capsaicin-induced apoptosis was observed when the cells were transfected by a non-targeting control-siRNA. (D) Both the TRPV6 siRNAs did not induce apoptosis (as measured by cleaved PARP ELISA experiments) in untreated DMS 53 and DMS 114 human SCLC cells. The absorbance of untransfected cell lysates was considered to be equal to 1 , and the absorbance values in all other samples were calculated as fold-increase relative to the control. Each sample was measured in duplicate. The entire experiment was done two independent times. Data represent mean \pm SEM. Results indicated by a different letter or an asterisk are significantly different $(P<0.05)$. 
(A)

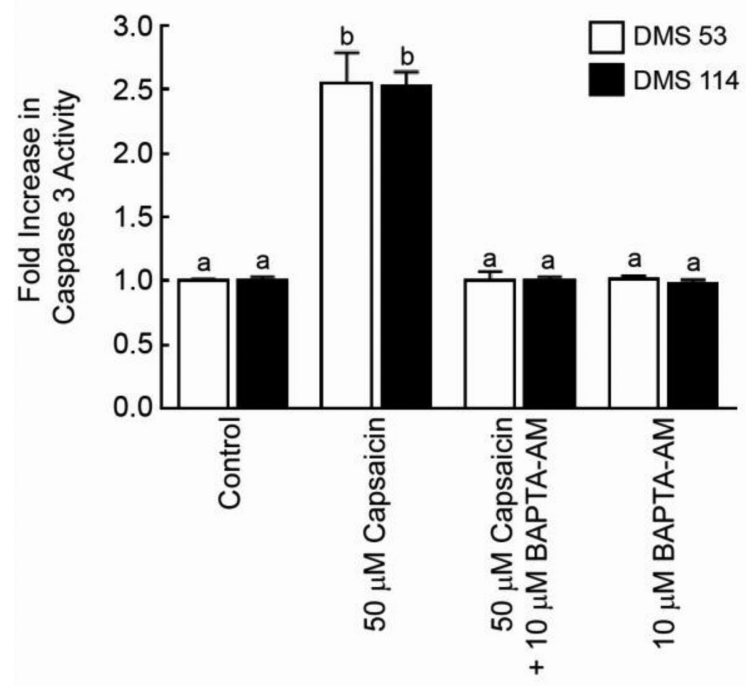

(C)

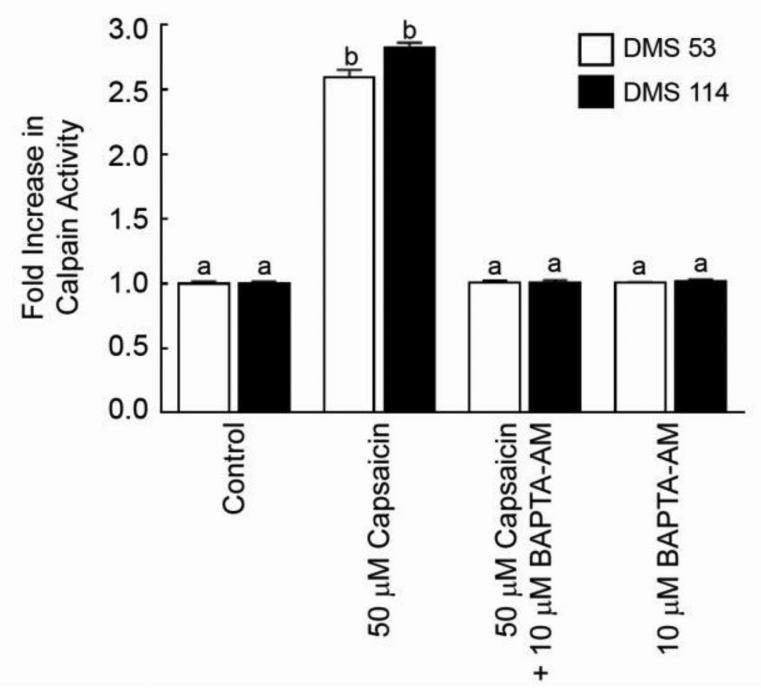

(B)

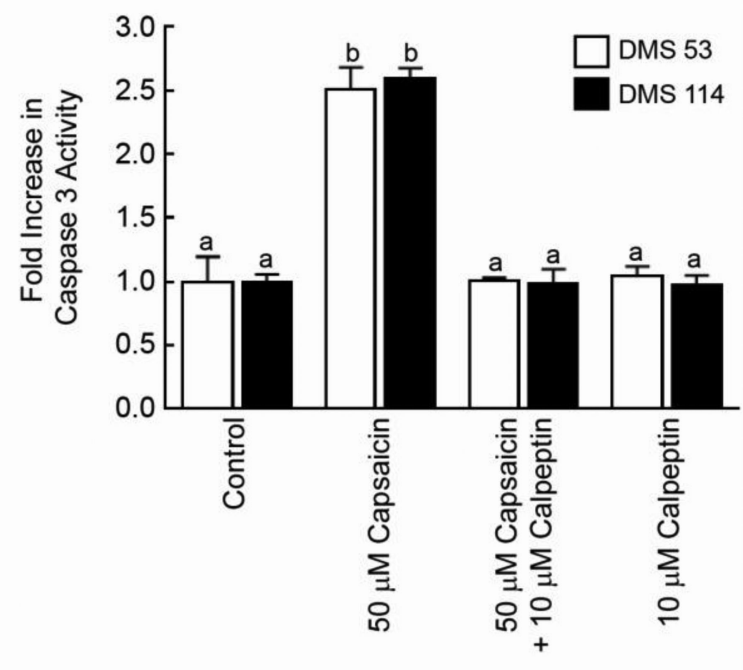

(D)

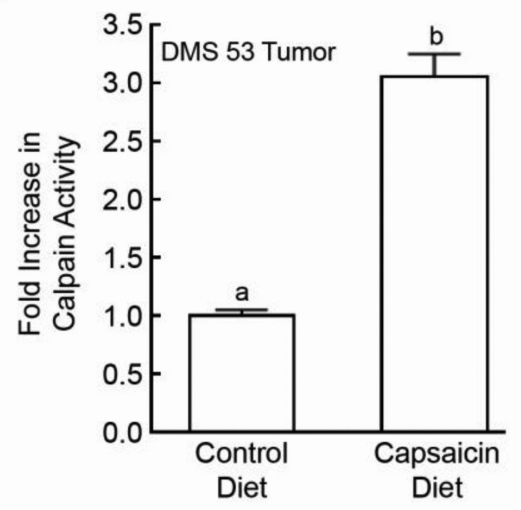

Fig. 5.

Caspase-3 activity assays reveal that capsaicin-induced apoptosis requires intracellular calcium and the calpain pathway. (A) The intracellular calcium chelator BAPTA-AM abrogated the pro-apoptotic activity of capsaicin. (B) The calpain inhibitor calpeptin reversed capsaicin-induced apoptosis in DMS 53 and DMS 114 cells. (C) Capsaicin increased the calpain activity in DMS 53 and DMS 114 human SCLC cells. Capsaicininduced calpain activity was blocked by the calcium chelator BAPTA-AM. Each sample was analyzed in duplicate and the experiment was completed two independent times. (D) Tumors lysates were prepared from nude mice experiments represented in Figure 2A. The lysates isolated from capsaicin-fed mice displayed higher calpain activity than tumor lysates 
from control untreated mice. Data represent mean \pm SEM. Results indicated by a different letter are significantly different $(P<0.05)$. 
(A)

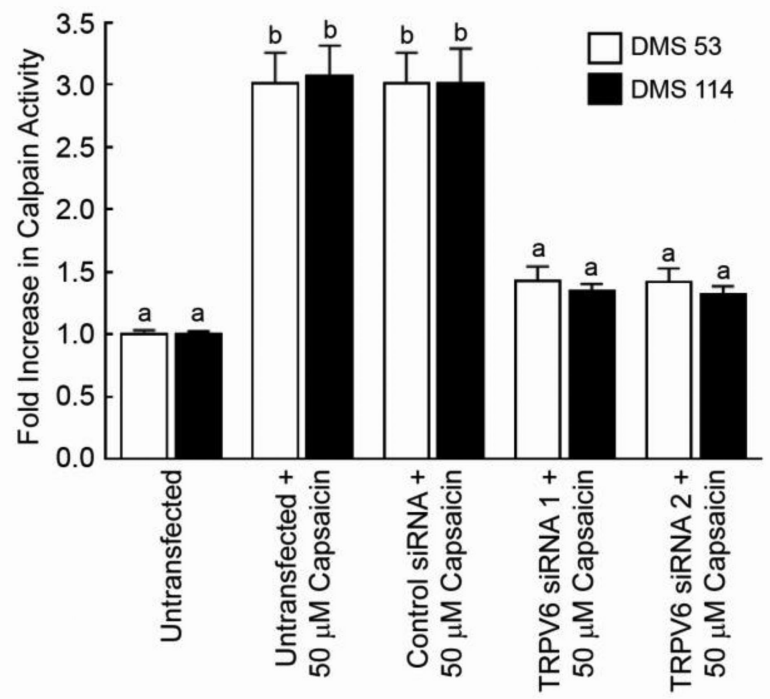

(B)

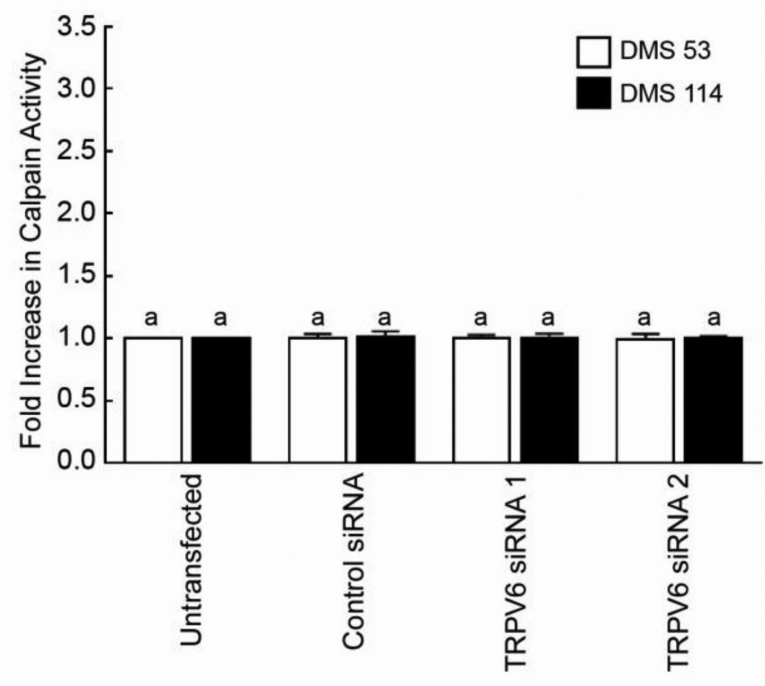

Fig. 6.

Capsaicin-induced upregulation of calpain activity was mediated by TRPV6 receptor. (A) The transfection of TRPV-6-siRNA (referred as TRPV6 siRNA 1) ablated capsaicininduced calpain activation in DMS 53 and DMS 114 cells. No effect was seen with a nontargeting control siRNA. The experiment was repeated with a second independent TRPV6 siRNA (referred as TRPV6 siRNA 2) and analogous results were obtained. (B) However, neither of the TRPV6 siRNA had any impact on calpain activity of untreated DMS 53 and DMS 114 human SCLC cells. Data represent mean \pm SEM. Results indicated by a different letter are significantly different $(P<0.05)$. 